\title{
Contagem de fótons no infravermelho próximo e médio via conversão de freqüências aplicada a comunicações quânticas
}

\author{
Guilherme Penello Temporão
}

Tese (Doutorado em Engenharia Elétrica). Pontifícia Universidade Católica do Rio de Janeiro. Rio de Janeiro, 2007. 


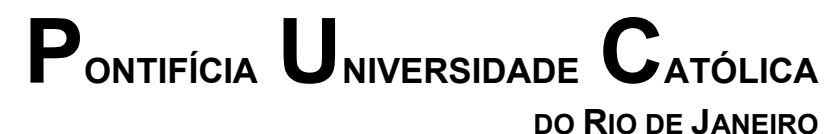

DO RIO DE JANEIRO

Guilherme Penello Temporão

Contagem de fótons no infravermelho próximo e médio via conversão de freqüências aplicada a comunicações quânticas

Tese de Doutorado

Tese apresentada como requisito parcial para obtenção do título de Doutor pelo Programa de Pós-Graduação em Engenharia Elétrica da PUC-Rio.

Orientador: Jean Pierre von der Weid

Rio de Janeiro, março de 2007 
Contagem de fótons no infravermelho próximo e médio via conversão de freqüências para comunicações quânticas

Tese apresentada como requisito parcial para obtenção do título de Doutor pelo Programa de Pós-Graduação em Engenharia Elétrica da PUC-Rio. Aprovada pela Comissão Examinadora abaixo assinada.

\author{
Jean Pierre von der Weid \\ Orientador \\ PUC-Rio \\ Nicolas Gisin \\ Co-orientador \\ Université de Genève \\ Isabel Cristina dos Santos Carvalho \\ PUC-Rio \\ Maria Cristina Ribeiro Carvalho \\ PUC-Rio \\ Rogério Passy \\ MLS Wireless \\ Paulo Henrique Souto Ribeiro \\ UFRJ \\ Alexandre de Oliveira Dal Forno \\ UERJ \\ José Eugenio Leal
}

Coordenador Setorial do Centro Técnico Científico - PUC-Rio

Rio de Janeiro, 5 de março de 2007 
Todos os direitos reservados. É proibida a reprodução total ou parcial do trabalho sem autorização da universidade, do autor e do orientador.

\section{Guilherme Penello Temporão}

Formado em Engenharia Elétrica com Ênfase em Telecomunicações pela PUC-Rio em 2002. Suas atuais áreas de interesse incluem metrologia em componentes ópticos, óptica quântica e teoria da informação quântica.

Ficha Catalográfica

Temporão, Guilherme Penello
Contagem de fótons no infravermelho próximo e
médio via conversão de freqüências aplicada a
comunicações quânticas / Guilherme Penello Temporão ;
orientador: Jean Pierre Von der Weid. - 2007.
199 f. : il. ; $30 \mathrm{~cm}$
Tese (Doutorado em Engenharia Elétrica)-
Pontifícia Universidade Católica do Rio de Janeiro, Rio de
Janeiro, 2007.
Inclui bibliografia.
1. Engenharia elétrica - Teses. 2. Contagem de
fótons. 3. Óptica não-linear. 4. Conversão de frequências.
5. Comunicações quânticas. 6. Criptografia quântica. I.
Weid, Jean Pierre von der. II. Pontifícia Universidade
Católica do Rio de Janeiro. Departamento de Engenharia
Elétrica. III. Título.

CDD: 621.3 


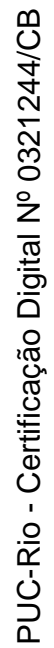

Para Ana. 


\section{Agradecimentos}

Aos profs. Jean Pierre von der Weid e Nicolas Gisin, pela sabedoria, orientação e principalmente pela oportunidade que me foi concedida.

Ao prof. Hugo Zbinden, meu $3^{\circ}$ orientador, que acompanhou de perto cada passo desta tese, dentro e fora do laboratório, por todas as discussões, idéias e sugestões.

Aos colegas da Universidade de Genebra Sébastien Tanzilli, Damien Stucki, Matthieu Legré, Thomas Ganz e Robert Thew, pelo companheirismo, por toda a ajuda prestada nas montagens experimentais e pela contagiante determinação e vontade de produzir resultados; ao técnico e amigo Claudio Barreiro, pela paciência, prestatividade, simpatia e excelência dos serviços prestados.

À minha mulher Ana, sem a qual essa tese não existiria, por literalmente ter largado tudo para ir viver em outro país, longe da família, do trabalho e da praia.

A meus pais Liliane e José, pela inspiração, exemplo e apoio que me foram (e continuam sendo) dados ao longo da vida; ao restante da minha família e a meus sogros Tania e Antonio que, assim como meus pais, estiveram presentes ao longo de toda a duração dos trabalhos experimentais na Suíça, apesar da distância.

Aos amigos Mario dal Poz, Regina Ungerer, Andreza e André Lupi, Jorge Ferreira e Cristiane Giovannini, entre muitos outros amigos queridos, que estarão para sempre indissociáveis desta tese e de minhas lembranças desses dois anos de vida em Genebra. Um agradecimento especial a Rafael Cordeiro Azevedo, pela amizade verdadeira e pela inestimável herança que deixou a mim.

Aos demais professores e colegas do GAP-Optique (Uni. Genève), do CETUC (PUC-Rio) e a todos aqueles que, de alguma forma, contribuíram para a conclusão desse trabalho. 


\section{Resumo}

Temporão, Guilherme Penello. Contagem de fótons no infravermelho próximo e médio via conversão de freqüências aplicada a comunicações quânticas. Rio de Janeiro, 2007. 199p. Tese de Doutorado - Departamento de Engenharia Elétrica, Pontifícia Universidade Católica do Rio de Janeiro.

Dois dispositivos de contagem de fótons únicos, sensíveis a comprimentos de onda no infravermelho próximo e médio, são propostos e experimentalmente investigados. Ambos utilizam uma técnica de dois estágios, composta de uma etapa inicial de conversão de freqüências em um cristal não-linear seguida de detecção por um fotodiodo avalanche de silício. Enquanto o primeiro projeto é voltado à detecção de fótons únicos a $1.55 \mu \mathrm{m}$ para comunicações quânticas via fibra óptica, usando um processo intra-cavidade, o segundo projeto prevê o desenvolvimento de um contador de fótons operando a $4.65 \mu \mathrm{m}$ para sistemas de espaço livre. Neste caso, um estudo de viabilidade para um sistema prático de criptografia quântica operando em um comprimento de onda no infravermelho médio é realizado. Os resultados mostram que, usando a tecnologia disponível na atualidade, tal sistema pode ser construído, embora sua utilidade se mostre restrita a enlaces possuindo certas condições meteorológicas específicas.

\section{Palavras-chave}

Contagem de fótons; Óptica não-linear; Conversão de freqüências; Comunicações quânticas; Criptografia quântica; Óptica de espaço livre. 


\section{Abstract}

Temporão, Guilherme Penello. Single photon counting in the near- and mid-infrared via frequency up-conversion applied to quantum communications. Rio de Janeiro, 2007. 199p. Tese de Doutorado Departamento de Engenharia Elétrica, Pontifícia Universidade Católica do Rio de Janeiro.

Two single photon counting devices, operating at near- and mid-infrared wavelengths, are introduced and experimentally investigated. Both use a twostage technique, comprised of an initial frequency up-conversion procedure inside a nonlinear crystal followed by a silicon avalanche photodiode. Whereas the first project consists on detection of single photons at $1.55 \mu \mathrm{m}$ for fiber-optic-based quantum communications, using a cavity-enhanced procedure, the second project envisions the development of a single-photon counter operating at $4.65 \mu \mathrm{m}$ for free-space systems. In this case, a feasibility study for a practical quantum key distribution system operating in a mid-infrared wavelength is performed. The results show that, using present-day technology, such a system can be constructed, albeit its usefulness would be restricted to operation under very specific weather conditions.

\section{Keywords}

Single photon counting; Nonlinear optics; Frequency up-conversion; Quantum communications; Quantum Key Distribution; Free-Space Optics. 


\section{Sumário}

1. Introdução

2. Comunicações Quânticas

18

2.1. Qubits

19

2.1.1. Representação de qubits 20

2.1.2. Múltiplos qubits 23

2.1.3. Emaranhamento 24

2.1.4. Estados mistos e a matriz de densidade 25

2.1.5. Implementações práticas 29

2.2. Geração de fótons únicos 33

2.2.1. Lasers atenuados $\quad 34$

2.2.2. Fonte de fótons anunciados 36

2.3. O canal quântico $\quad 37$

2.4. Detecção de fótons únicos $\quad 40$

2.4.1. Caracterização de um contador de fótons 41

2.4.2. A dependência do comprimento de onda 46

2.5. Criptografia quântica 48

2.5.1. O problema da distribuição de chaves 49

2.5.2. Codificação em bases não-ortogonais 51

2.5.3. O protocolo BB84 54

2.5.4. Implementação prática $\quad 58$

3. Meios não-lineares e conversão de freqüências 67

3.1. Efeitos não-lineares de segunda ordem 70

3.2. Propriedades da susceptibilidade não-linear 72

3.2.1. Simetrias Espaciais 74

3.2.2. Coeficiente não-linear efetivo ( $\left.\mathrm{d}_{\mathrm{eff}}\right) \quad 76$

3.3. A equação de onda para meios não-lineares 77

3.3.1. As equações de onda acopladas para SFG 79

3.3.2. As Relações de Manley-Rowe 81 
3.3.3. Uma primeira expressão para a onda gerada 82

3.3.4. O efeito de depleção do sinal de entrada 85

3.4. Casamento de fase $\quad 89$

3.4.1. Casamento de fase via birrefringência 91

3.4.2. Quasi-Casamento de Fase 96

3.5. A Teoria de Boyd-Kleinman para Feixes Gaussianos 99

3.5.1. Análise para SHG 100

3.5.2. Análise para SFG 106

3.6. Geração de Soma de Freqüências de um ponto de vista quântico 108

3.6.1. Considerações fundamentais 108

3.6.2. Eficiência quântica de conversão 110

3.6.3. Descrição quântica do processo de SFG 112

4. Contagem de Fótons a $1.55 \mu \mathrm{m}$ via SFG Intra-Cavidade $\quad 114$

4.1. Primeira tentativa: cavidade linear simétrica 115

4.1.1. Análise de estabilidade e eficiência de conversão 117

4.1.2. O problema da focalização 120

4.1.3. Montagem experimental 124

4.2. A cavidade em "L" 132

4.2.1. Considerações geométricas e critério de estabilidade 133

4.2.2. Resultados Experimentais 136

4.3. Discussão dos resultados 140

5. Contagem de fótons a $4.65 \mu \mathrm{m}$ para criptografia quântica via canal atmosférico 144

5.1. Seleção do comprimento de onda 146

5.1.1. Limites impostos pelo processo de detecção 147

5.1.2. Ruído térmico 148

5.1.3. Condições atmosféricas e o espalhamento Mie 152

5.2. Resultados experimentais 155

5.2.1. Obtenção do sinal de soma de freqüências 155

5.2.2. Medidas de eficiência e ruído no regime de contagem de fótons 160

5.2.3. Desempenho do detector 164 
5.2.4. Montagem experimental para avaliação da QBER

5.3. Estudo de viabilidade para criptografia quântica

5.3.1. Construindo Alice e Bob

5.3.2. Desempenho e limites no canal atmosférico

5.3.3. O efeito da luz solar: uma discussão qualitativa

6 . Conclusão

7 . Referências bibliográficas

Apêndice A O que é um Espaço de Hilbert? 


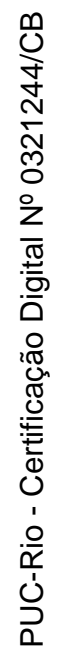




\section{Lista de figuras}

Figura 1: Representação de qubits como pontos na superfície de uma esfera.

Figura 2. Preparação e medida de qubits codificados em polarização 31

Figura 3. Preparação e medida de qubits codificados em time-bins 32

Figura 4. Esquema simplificado para fonte de fótons "anunciados" 36

Figura 5. Evolução de sistemas quânticos isolados e abertos 38

Figura 6. Sistema de criptografia quântica, no qual Alice e Bob estão interligados por um canal quântico e um canal clássico.

Figura 7. (a) Distribuição de cargas em átomo neutro; (b) A nuvem eletrônica se desloca com a aplicação de um campo elétrico externo.

Figura 8. Efeitos não-lineares de segunda ordem. (a) SHG; (b) Retificação óptica; (c) SFG; (d) DFG 71

Figura 9. Função $\operatorname{sinc}^{2}(x)=\operatorname{sen}^{2}(x) / x^{2}$

Figura 10. Variação da intensidade da onda de soma de freqüências e do sinal de entrada em função do comprimento do cristal não-linear $\quad 88$ Figura 11. O conceito de casamento de fase 89 Figura 12. Casamento de fase birrefringente 91 Figura 13. (a) Interação do tipo I, na qual os sinais de entrada possuem a mesma polarização. (b) Interação do tipo II, na qual os sinais de entrada possuem estados de polarização ortogonais. $\quad 92$

Figura 14. Casamento de fase por ângulo 94

Figura 15. (a) Fasores em fase $(\Delta k=0)$; (b) Soma de fasores com $\Delta k \neq 0$ para $x=I_{c} ;$ (c) Idem para $x=2 I_{c}$

Figura 16. Quasi-casamento de fase 98

Figura 17. Focalização forte e fraca de feixes gaussianos 99

Figura 18. Análise de Boyd-Kleinman para feixes gaussianos. 101

Figura 19. Fator de focalização de Boyd-Kleinman em função do grau de focalização para diferentes valores de B. 105

Figura 20. Os valores de $\xi_{1 m}$ e $\xi_{2 m}$ para diferentes valores de $k$, em função de $B$. 
Figura 21. Valor máximo do fator de focalização em função de $k$ e $B \quad 107$

Figura 22. Diagrama de energia para o processo de SFG 109

Figura 23. Cavidade ressonante utilizando dois espelhos esféricos

Próximo do espelho da direita é posicionado o cristal laser (LC), e próximo ao centro da cavidade encontra-se o cristal não-linear (NC) 116

Figura 24. Dependência do tamanho efetivo ideal da cavidade com o fator de focalização

Figura 25. Desenho mostrando as distâncias envolvidas no cálculo da posição e distância focal da lente

Figura 26. Distância focal mínima (normalizada pelo raio de curvatura do espelho) em função do comprimento efetivo normalizado.

Figura 27. Montagem experimental usando cavidade linear 125

Figura 28. Imagem do feixe a $1064 \mathrm{~nm}$ na saída da cavidade 126

Figura 29. Diminuição da potência do laser $\mathrm{YVO}_{4}$ com o aumento do comprimento da cavidade

Figura 30. Potência do sinal convertido em função da potência de bombeio

Figura 31. Taxa de ruído em função da potência intra-cavidade a $1064 \mathrm{~nm}$.

Figura 32. Cavidade em "L"

Figura 33. Comportamento da posição da cintura e da distância de Rayleigh em função do comprimento da cavidade em "L"

Figura 34. Comportamento linear da potência intra-cavidade em função do bombeio para a configuração em "L"

Figura 35. Eficiência de conversão em função da potência intra-cavidade para a configuração em "L"

Figura 36. Ruído obtido na cavidade em "L"

Figura 37. Primeiros resultados obtidos pelo grupo de pesquisadores Haifeng Pan et al

Figura 38. Resultados recentes do grupo de Haifeng Pan et al

Figura 39. Curva típica de transmissão atmosférica sob condições climáticas limpas 
Figura 40. Linha sólida: probabilidade de ruído de fundo por pulso, em função do comprimento de onda. Linha tracejada: radiação solar normalizada, expressa em fótons por unidade de tempo por unidade de área por unidade de ângulo sólido.

Figura 41. Dados experimentais de atenuação atmosférica para um enlace de visibilidade variável sob efeito de névoa "coastal upslope"

Figura 42. Montagem experimental para medida clássica de eficiência de conversão.

Figura 43. Espectro do sinal convertido usando AGS e PPLN

Figura 44. Variação do sinal de soma de freqüências com a temperatura do cristal PPLN

Figura 45. Transmissão da luz através de $1 \mathrm{~cm}$ de cristal de niobato de lítio, em função do comprimento de onda 162

Figura 46. Variação teórica da sensibilidade com a eficiência global de detecção para a detecção via conversão de freqüências.

Figura 47. Montagem experimental para avaliação da QBER 167

Figura 48. Rotação de polarização usando dois polarizadores 168

Figura 49. Histograma do tempo de chegada dos fótons com relação ao tempo de emissão dos pulsos elétricos no QCL após vários segundos

Figura 50. Curva de visibilidade para detecção de fótons únicos a

$4.65 \mu \mathrm{m}$ na base horizontal-vertical para $\mu=0.8$.

Figura 51. Implementação prática do sistema de transmissão (Alice) 171

Figura 52. Implementação prática do sistema de recepção (Bob): primeira opção 172

Figura 53. Configuração alternativa para o sistema de Bob

Figura 54. Modelagem dos efeitos da radiação solar sobre um sistema de comunicações quânticas de espaço livre.

Figura 55. Função de fase de Mie para névoa de radiação com raio médio de $1 \mu \mathrm{m}$ para os comprimentos de onda relevantes 
Alice laughed: "There's no use trying," she said; "one can't believe impossible things."

"I daresay you haven't had much practice," said the Queen. "When I was younger, I always did it for half an hour a day. Why, sometimes I've believed as many as six impossible things before breakfast."

- Lewis Carroll, "Through the Looking Glass" 